\title{
A Parallel Gauss-Seidel Algorithm for Sparse Power System Matrices
}

\author{
D. P. Koester, S. Ranka, and G. C. Fox \\ School of Computer and Information Science and \\ The Northeast Parallel Architectures Center (NPAC) \\ Syracuse University \\ Syracuse, NY 13244-4100 \\ dpk@npac.syr.edu,ranka@top.cis.syr.edu,gcf@npac.syr.edu
}

\begin{abstract}
We describe the implementation and performance of an efficient parallel Gauss-Seidel algorithm that has been developed for irregular, sparse matrices from electrical power systems applications. Although, GaussSeidel algorithms are inherently sequential, by performing specialized orderings on sparse matrices, it is possible to eliminate much of the data dependencies caused by precedence in the calculations. A twopart matrix ordering technique has been developed - first to partition the matrix into block-diagonalbordered form using diakoptic techniques and then to multi-color the data in the last diagonal block using graph coloring techniques. The ordered matrices often have extensive parallelism, while maintaining the strict precedence relationships in the Gauss-Seidel algorithm. We present timing results for a parallel Gauss-Seidel solver implemented on the Thinking Machines CM-5 distributed memory multi-processor. The algorithm presented here requires active message remote procedure calls in order to minimize communications overhead and obtain good relative speedup.
\end{abstract}

\section{Introduction}

Power system distribution networks are generally hierarchical with limited numbers of high-voltage lines transmitting electricity to highly interconnected local networks that eventually distribute power to customers. Electrical power grids have graph representations which in turn can be expressed as matrices - electrical buses are graph nodes and matrix diagonal elements, while electrical transmission lines are graph edges which can be represented as non-zero offdiagonal matrix elements.
We show that it is possible to identify the hierarchical structure within a power system matrix using only the knowledge of the interconnection pattern by tearing the matrix into partitions and coupling equations that yield a block-diagonal-bordered matrix. Nodetearing-based partitioning identifies the basic network structure that provides parallelism for the majority of calculations within a Gauss-Seidel iteration. Graph multi-coloring has been used to order the last diagonal matrix block and subsequently identify available parallelism. We implemented explicit load balancing as part of each of the aforementioned ordering steps to maximize parallel algorithm efficiency.

We implemented the parallel Gauss-Seidel algorithm on the Thinking Machines CM-5 distributed memory multi-processor exclusively using explicit message passing based on Connection Machine active message layer (CMAML) remote procedure calls (RPCs). The communications paradigm we use throughout this algorithm employs CMAML RPCs to send individual values to destination processors as soon as values have been calculated. This paradigm greatly simplified the development and implementation of this parallel sparse Gauss-Seidel algorithm.

Parallel implementations of Gauss-Seidel have generally been developed for regular problems such as the solution of Laplace's equations by finite differences $[4,5]$, where red-black coloring schemes are used to provide independence in the calculations and some parallelism. This scheme has been extended to multicoloring for additional parallelism in more complicated regular problems [5], however, we are interested in the solution of irregular linear systems. There has been some research into applying parallel Gauss-Seidel to circuit simulation problems [11], although this work showed poor parallel speedup potential in a theoretical study. This reference also extended traditional Gauss- 
Seidel and Gauss-Jacobi techniques to waveform relaxation methods that trade overhead and convergence rate for parallelism. Other research with parallel Gauss-Seidel methods for power systems applications is presented in [7], although our research differs substantially from that work: our research utilizes a different matrix ordering paradigm, a different load balancing paradigm, and a different parallel implementation paradigm. Our work utilizes diakoptic-based matrix partitioning techniques developed initially for a parallel block-diagonal-bordered direct sparse linear solver $[9,10]$. In reference [9] we examined load balancing issues associated with partitioning power systems matrices for parallel Choleski factorization.

The paper is organized as follows. In section 2, we introduce the electrical power systems application that is the basis for this work. In section 3, we briefly review the Gauss-Seidel iterative method, then present a theoretical derivation of the available parallelism with Gauss-Seidel for a block-diagonal-bordered form sparse matrix. We discuss the preprocessing phase that orders the sparse matrices in section 5 , and we describe our parallel Gauss-Seidel algorithm implementation in section 6 . Analysis of parallel algorithm performunce for actual power system load flow matrices are presented in section 7 . We present our conclusions in section 8 .

\section{Power System Applications}

The underlying motivation for our research is to improve the performance of electrical power system applications to provide real-time power system control and real-time support for proactive decision making. This research has focused on matrices from load-flow applications [14]. Load-flow analysis examines steadystate equations based on the symmetric positive definite network admittance matrix that represents the power system distribution network. Load flow analysis entails the solution of non-linear systems of simultaneous equations, which are performed by repeatedly solving sparse linear equations. Sparse linear solvers account for the majority of floating point operations encountered in load-flow analysis.

\section{The Gauss-Seidel Method}

We are considering an iterative solution to the linear system

$$
\mathbf{A x}=\mathbf{b},
$$

where $\mathbf{A}$ is an $(n \times n)$ sparse matrix, $\mathbf{x}$ and $\mathbf{b}$ are vectors of length $n$, and we are solving for $\mathbf{x}$. Iterative solvers are an alternative to direct methods that attempt to calculate an exact solution to the system of equations. Iterative methods attempt to find a solution to the system of linear equations by repeatedly solving the linear system using approximations to the $\mathbf{x}$ vector. Iterations continue until the solution is within a predetermined acceptable bound on the error.

The Gauss-Seidel method can be written as:

$$
x_{i}^{(k+1)}=\frac{1}{a_{i i}}\left(b_{i}-\sum_{j<i} a_{i j} x_{j}^{(k+1)}-\sum_{j>i} a_{i j} x_{j}^{(k)}\right),
$$

where:

$x_{i}^{(k)}$ is the $i^{\text {th }}$ unknown in $\mathbf{x}$ during the $k^{\text {th }}$ iteration, $i=1, \cdots, n$ and $k=0,1, \ldots$,

$x_{i}^{(0)}$ is the initial guess for the $i^{\text {th }}$ unknown in $\mathbf{x}$,

$a_{i j}$ is the coefficient of $\mathbf{A}$ in the $i^{t h}$ row and $j^{\text {th }}$ column,

$b_{i}$ is the $i^{t h}$ value in $\mathbf{b}$.

or

$$
\mathbf{x}^{(k+1)}=(\mathbf{D}+\mathbf{L})^{-1}\left[\mathbf{b}-\mathbf{U} \mathbf{x}^{(k)}\right]
$$

where:

$\mathbf{x}^{(k)}$ is the $k^{\text {th }}$ solution to $\mathbf{x}, k=0,1, \ldots$,

$\mathbf{x}^{(0)}$ is the initial guess at $\mathbf{x}$,

$\mathbf{D}$ is the diagonal of $\mathbf{A}$,

$\mathbf{L}$ is the strictly lower triangular portion of $\mathbf{A}$,

$\mathbf{U}$ is the strictly upper triangular portion of $\mathbf{A}$,

$\mathbf{b}$ is right-hand-side vector.

The representation in equation 2 is used in the development of the parallel algorithm, while the equivalent matrix-based representation in equation 3 is used below in discussions of available parallelism. We present a general sequential sparse Gauss-Seidel algorithm in figure 1. This algorithm calculates a constant number of iterations before checking convergence.

It is very difficult to determine if one-step iterative methods, like the Gauss-Seidel method, converge for general matrices. Nevertheless, it is possible to prove that the Gauss-Seidel method does converge and yields the unique solution $\mathbf{x}$ for $\mathbf{A x}=\mathbf{b}$ with any initial starting vector $\mathbf{x}^{(\mathbf{0})}$ for both diagonally dominant and symmetric positive definite matrices [5]. These theorems prove that the Gauss-Seidel method converges for these matrix types, however, there is no evidence as to the rate of convergence.

Symmetric sparse matrices can be represented by graphs with elements in equations corresponding to 


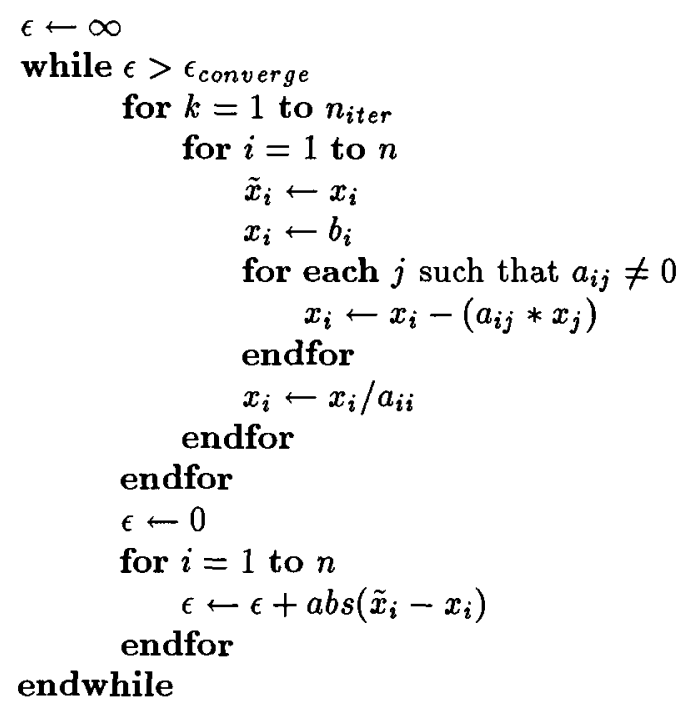

Figure 1: Sparse Gauss-Seidel Algorithm

undirected edges in the graph [6]. Ordering a symmetric sparse matrix is actually little more than changing the labels associated with nodes in an undirected graph. Modifying the ordering of a sparse matrix is simple to perform using a permutation matrix $\mathbf{P}$ that simply generates elementary row and column exchanges. Applying a permutation matrix to the original linear system in equation 1 yields

$$
\left(\mathbf{P A P} \mathbf{P}^{T}\right)(\mathbf{P x})=(\mathbf{P b}) .
$$

While ordering the matrix can greatly simplify accessing parallelism inherent within the matrix structure, ordering can have an effect on convergence [5]. In section 7 , we present empirical data to show that in spite of the ordering to yield parallelism, convergence appears to be rapid for positive definite power systems load-flow matrices.

\section{Available Parallelism}

While Gauss-Seidel algorithms for dense matrices are inherently sequential, it is possible to identify sparse matrix partitions that do not have mutual data dependencies, so calculations can proceed in parallel while maintaining the strict precedence rules in the Gauss-Seidel technique. Entire sparse matrix partitions can be calculated in parallel without requiring communications. All parallelism in the Gauss-Seidel algorithm is derived from within the actual interconnection relationships between elements in the matrix.
While much of the parallelism in this algorithm comes from the block-diagonal-bordered ordering of the sparse matrix, further ordering of the last diagonal block is required to provide parallelism in what would otherwise be a purely sequential portion of the algorithm. The last diagonal block represents the interconnection structure within the equations that couple the partitions in the block-diagonal portion of the matrix. These equations are rather sparse, so, it is simple to color the graph representing this portion of the matrix. Separate graph colors represent rows where $\mathbf{x}^{(k+1)}$ can be calculated in parallel, because within a color, no two nodes have any adjacent edges.

To clearly identify the available parallelism in the block-diagonal-bordered Gauss-Seidel method, we define a block diagonal matrix partition, apply that partition to formula 3, and equate terms to identify available parallelism. We must also define a subpartitioning of the last diagonal block to identify parallelism after multi-coloring.

\subsection{Block-Diagonal-Bordered Matrices}

We define a partitioning of the system of linear equations defined in equation 4 , where the permutation matrix $\mathbf{P}$ orders the matrix into block-diagonalbordered form. We define

$$
\mathbf{P A P}^{T}=\left(\begin{array}{cccc}
\mathbf{A}_{1,1} & \mathbf{0} & & \mathbf{A}_{1, m} \\
\mathbf{0} & \ddots & & \vdots \\
& & \mathbf{A}_{m-1, m-1} & \mathbf{A}_{m-1, m} \\
\mathbf{A}_{m, 1} & \cdots & \mathbf{A}_{m, m-1} & \mathbf{A}_{m, m}
\end{array}\right),
$$

and $\mathbf{P x}$ and $\mathbf{P b}$ are partitioned with similar dimensions. Equation 3 divides the $\mathbf{P A} \mathbf{P}^{T}$ matrix into a diagonal component $\mathbf{D}$, a strictly lower diagonal matrix $\mathbf{L}$, and a strictly upper diagonal matrix $\mathbf{U}$ such that:

$$
\mathbf{P A} \mathbf{P}^{T}=\mathbf{D}+\mathbf{L}+\mathbf{U}
$$

Derivation of the block-diagonal-bordered form of the $\mathbf{D}, \mathbf{L}$, and $\mathbf{U}$ matrices is straightforward. Equation 3 requires the calculation of $(\mathbf{D}+\mathbf{L})^{-1}$, which also is simple to determine explicitly, because this matrix has block-diagonal-lower-bordered form.

Given these partitioned matrices, it is relatively straightforward to identify available parallelism. For $i,(i=1, \cdots, m-1)$, we obtain:

$$
\mathbf{x}_{i}^{(k+1)}=\left(\mathbf{D}_{i, i}+\mathbf{L}_{i, i}\right)^{-1}\left[\mathbf{b}_{i}-\mathbf{U}_{i, i} \mathbf{x}_{i}^{(k)}-\mathbf{U}_{i, m} \mathbf{x}_{m}^{(k)}\right]
$$


and for the lower border and last diagonal block we obtain:

$$
\mathbf{x}_{m}^{(k+1)}=\frac{\left(\mathbf{D}_{m, m}+\mathbf{L}_{m, m}\right)^{-1} \times}{\left[\mathbf{b}_{m}-\sum_{i=1}^{m-1}\left(\mathbf{L}_{m, i}^{-1} \mathbf{x}_{i}^{(k+1)}\right)-\mathbf{U}_{m, m} \mathbf{x}_{m}^{(k)}\right]}
$$

We can identify the parallelism in the blockdiagonal-bordered portion of the matrix by examining equations 7 and 8 . If the block-diagonal-bordered matrix partitions $\mathbf{A}_{i, i}, \mathbf{A}_{m, i}$, and $\mathbf{A}_{i, m}(1 \leq i \leq m-1)$ are assigned to the same processor, then there are no communications until $\mathbf{x}_{m}^{(k+1)}$ is calculated. Note that the vector $\mathbf{x}_{m}^{(k)}$ is required for the calculations in each partition, however, there is no violation of the strict precedence rules in the Gauss-Seidel method, because these values are not calculated until the last step. After calculating $\mathbf{x}_{i}^{(k+1)}$ in the first $(m-1)$ partitions, the values of $\mathbf{x}_{m}^{(k+1)}$ must be calculated using the lower border and last block. If we assign

$$
\hat{\mathbf{b}}=\mathbf{b}_{m}-\sum_{i=1}^{m-1}\left(\mathbf{L}_{m, i}^{-1} \mathbf{x}_{i}^{(k+1)}\right),
$$

then the formulation of $\mathbf{x}_{m}^{(k+1)}=\hat{\mathbf{x}}^{(k+1)}$ looks similar to equation 3 :

$$
\hat{\mathbf{x}}^{(k+1)}=\left(\mathbf{D}_{m, m}+\mathbf{L}_{m, m}\right)^{-1}\left[\hat{\mathbf{b}}-\mathbf{U}_{m, m} \mathbf{x}^{(k)}\right] .
$$

Figure 2 describes the calculation steps in the parallel Gauss-Seidel for a block-diagonal-bordered sparse matrix. This figure depicts four diagonal blocks, and data/processor assignments (P1, P2, P3, and P4) are listed for the data block.

\subsection{Multi-Colored Matrices}

The ordering imposed by the permutation matrix $\mathbf{P}$, includes multi-coloring-based ordering of the last diagonal block that produces sub-partitions with parallelism, We define the sub-partitioning as:

$$
\mathbf{A}_{m, m}=\left(\begin{array}{cccc}
\hat{\mathbf{D}}_{1,1} & \hat{\mathbf{A}}_{1,2} & \cdots & \hat{\mathbf{A}}_{1, c} \\
\hat{\mathbf{A}}_{2,1} & \hat{\mathbf{D}}_{2,2} & \cdots & \hat{\mathbf{A}}_{2, c} \\
\vdots & & \ddots & \vdots \\
\hat{\mathbf{A}}_{c, 1} & \hat{\mathbf{A}}_{c, 2} & \cdots & \hat{\mathbf{D}}_{c, c}
\end{array}\right)
$$

where $\hat{\mathbf{D}}_{i, i}$ are diagonal blocks and $c$ is the number of colors. After forming $\mathbf{L}_{m, m}$ and $\mathbf{U}_{m, m}$, it is straight forward to prove that:

$$
\hat{\mathbf{x}}_{i}^{(k+1)}=\hat{\mathbf{D}}_{i, i}^{-1}\left[\hat{\mathbf{b}}_{i}-\sum_{j<i} \hat{\mathbf{A}}_{i, j} \hat{\mathbf{x}}_{j}^{(k+1)}-\sum_{j>i} \hat{\mathbf{A}}_{i, j} \hat{\mathbf{x}}_{j}^{(k)}\right]
$$

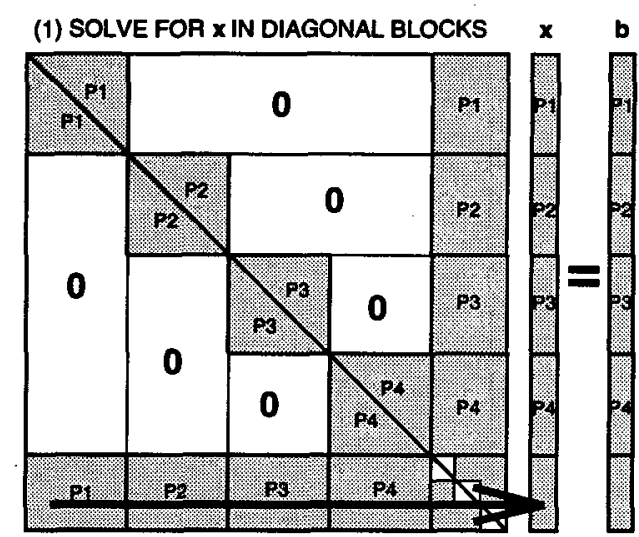

(2) CALCULATE (MATRIXX VECTOR) (3) SOLVE FOR $x$ PRODUCT AND SEND IN LAST

DIAGONAL BLOCK

Figure 2: Block-Bordered-Diagonal Form GaussSeidel Method - $(m=5)$

Calculating $\hat{\mathbf{x}}_{i}^{(k+1)}$ in each sub-partition (color) of the last diagonal block does not require values of $\hat{\mathbf{x}}_{i}^{(k+1)}$ within the sub-partition, so we can calculate the individual values within a color in any order and distribute these calculations to separate processors without concern for precedence. In order to maintain the strict precedence in the Gauss-Seidel algorithm, the values of $\hat{\mathbf{x}}_{i}^{(k+1)}$ calculated in each step must be broadcast to all processors, and processing cannot proceed for any processor until it receives the new values of $\hat{\mathbf{x}}_{i}^{(k+1)}$ from all other processors. Figure 3 illustrates the data/processor assignments in the last diagonal block.

\section{The Preprocessing Phase}

In the previous section, we developed the theory for parallel Gauss-Seidel methods, however, before such techniques can be implemented on real power systems matrices, we must be able to generate the permutation matrices, $\mathbf{P}$, to produce block-diagonalbordered/multi-colored sparse matrices. All available parallelism for our Gauss-Seidel algorithm is identified from the interconnection structure of elements in the sparse matrix during this preprocessing phase. Inherent in both preprocessing steps is explicit load-balancing to determine processor/data mappings for efficient implementation of the Gauss-Seidel algorithm. This preprocessing phase incurs significantly more overhead than solving a single instance of the 


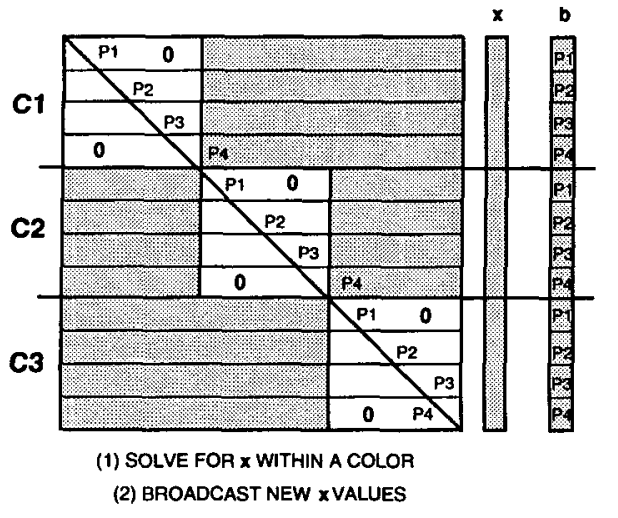

Figure 3: Multi-Colored Gauss-Seidel Method for the Last Diagonal Block - $(c=3)$

sparse matrix; consequently, the use of this technique will be limited to problems that have static matrix structures that can reuse the ordered matrix multiple times in order to amortize the cost of the preprocessing phase over numerous matrix solutions.

\subsection{Ordering the Matrix into Block- Bordered-Diagonal Form}

We require a technique that orders irregular matrices into block-diagonal-bordered form while limiting the number of coupling equations. Minimizing the number of coupling equations minimizes the size of the last diagonal block, and minimizes the amount of broadcast communications required when calculating values of $\hat{\mathbf{x}}^{(k+1)}$. Minimizing the size of the last diagonal block has some drawbacks. We have found an inverse relationship between last block size and loadimbalance between processors. This can affect potential parallelism if the resulting workload in the diagonal blocks cannot be distributed uniformly throughout a multi-processor [9]. When determining the optimal ordering for a sparse matrix, the size of the last diagonal block and the subsequent additional communications may be traded for an ordering that yields good load balance in the highly parallel portion of the calculations, especially for larger numbers of processors.

We have chosen node-tearing $[10,12]$, which is a specialized form of diakoptics, to order sparse power systems matrices into block-diagonal-bordered form. We have selected node-tearing nodal analysis because this algorithm determines the natural structure in the matrix while providing the means to minimize the number of coupling equations [12]. Tearing here refers to breaking the original problem into smaller subproblems whose partial solutions can be combined to give the solution of the original problem.

The node-tearing-based ordering algorithm has à user-selectable input parameter, $\max _{D B}$, the maximum size of the diagonal blocks. Varying this input parameter permits the user to vary characteristics in the ordered diagonal blocks. Empirical data is presented later in section 7 to illustrate parallel linear solver algorithm performance as a function of this parameter.

Load balancing for node-tearing-based ordering is performed with a simple pigeon-hole type algorithm that uses a metric based on the number of floating point multiply/add operations in a partition, instead of simply using the number of rows per partition. Load balancing examines the number of operations when calculating $\mathbf{x}^{(k+1)}$ in the matrix partitions and the number of operations when calculating the sparse matrix vector products in preparation to solve for $\hat{\mathbf{x}}^{(k+1)}$. This algorithm finds an optimal distribution for workload to processors, however, actual disparity in processor workload is dependent on the actual irregular sparse matrix structure.

\subsection{Ordering the Last Diagonal Block}

The multi-coloring algorithm we selected for this work is based on the saturation degree ordering algorithm [1]. We also require load balancing, a feature not commonly implemented within graph multicoloring. The saturation degree ordering algorithm selects a node in the graph that has the largest number of differently colored neighbors. We have added the capability to the saturation degree ordering algorithm to select the color for a node in a manner that equalizes the number of nodes with a particular color. The graphs encountered for coloring in this work were very sparse, and often required three or less colors. Details of this graph multi-coloring algorithm are presented in $[8]$.

\section{Parallel Implementation}

We have implemented a parallel version of a blockdiagonal-bordered sparse Gauss-Seidel algorithm in the $\mathrm{C}$ programming language for the Thinking Machines CM-5 multi-computer using CMAML RPCs as the exclusive basis for interprocessor communications [13]. Underlying the whole concept of active messages is the paradigm that the user takes the responsibility for handling messages as they arrive at a destination. 
The user writes a handler function that takes the data from a register and uses it in a calculation or assigns the data to memory. By assigning message handling responsibilities to the user, communications overhead can be significantly reduced.

Significant improvements in the performance of the algorithm were observed for active messages, when compared to more traditional communications paradigms that use the standard blocking CMMD_send and CMMD receive functions in conjunction with packing data into communications buffers. A significant portion of communications require each processor to send short data buffers to every other processor. For traditional message passing paradigms, the cost for communications increases drastically as the number of processors increases, because each message incurs the same latency regardless of the amount of data sent. As a result, performance for buffered communications quickly becomes unacceptable as the number of processors increases.

To significantly reduce communications overhead, we implemented each portion of the algorithm using CMAML remote procedure calls (CMAML $r p c$ ). The communications paradigm we use throughout this algorithm is to send a double precision data value to the destination processor as soon as the value is calculated. Communications in the algorithm occur at distinct time phases, so polling for the active message handler function is efficient. An active message on the CM-5 has a four word payload, which is more than adequate to send a double precision floating point value and an integer vector position indicator. The use of active messages greatly simplified the development and implementation of this parallel sparse Gauss-Seidel algorithm, because there was no requirement to maintain and pack communications buffers.

This implementation uses implicit data structures based on vectors of $\mathrm{C}$ programming language structures to store and retrieve data efficiently within the sparse matrix. These data structures provide good cache coherence, because non-zero data values and column location indicators are stored in adjacent physical memory locations. Data is stored as sparse vectors with implicit referencing, so only the SPARC processors on each node were used for calculations.

Our parallel Gauss-Seidel algorithm has the following distinct sections:

1. solve for $\mathbf{x}^{(k+1)}$ in the diagonal blocks

2. calculate $\hat{\mathbf{b}}=\mathbf{b}_{m}-\sum_{i=1}^{m-1}\left(\mathbf{L}_{m, i}^{-1} \mathbf{x}_{i}^{(k+1)}\right)$ by forming the (matrix $\times$ vector) products in parallel

3. solve for $\hat{\mathbf{x}}^{(k+1)}$ in the last diagonal block

\section{4. check convergence}

A pseudo-code representation of the parallel GaussSeidel solver is presented in figure 4 .

\section{Empirical Results}

Overall performance of our parallel Gauss-Seidel linear solver is dependent on both the performance of the matrix ordering in the preprocessing phase and the performance of the parallel Gauss-Seidel implementation. Because these two components of the parallel Gauss-Seidel implementation are inextricably related, the best way to assess the potential of this technique is to measure the speedup performance using real power system load-flow matrices. We first present speedup results for three separate power systems matrices:

- BCSPWR09 - 1,723 nodes and 2,394 edges [2]

- BCSPWR10 - 5,300 nodes and 8,271 edges [2]

- EPRI-6K - 4,180 nodes and 5,226 edges [3]

Matrices BCSPWR09 and BCSPWR10 are from the Boeing Harwell series and the EPRI-6K matrix is distributed with the Extended Transient-Midterm Stability Program (ETMSP) from EPRI. These matrices have been preprocessed using a sequential program that orders the matrix, load balances each ordering step, and produces the implicit data structures for the parallel Gauss-Seidel linear solver. The preprocessing was repeated for multiple values of $\max _{D B}$, the input value to the node-tearing algorithm. Due to the static nature of the power system grid, such orderings could be reused for many hours or even days of calculations in real electrical power utility operations load-flow applications.

Empirical performance data was collected for each of the aforementioned power systems matrices using 1 through 32 processors on the Thinking Machines CM5 at the Northeast Parallel Architectures Center at Syracuse University. The NPAC CM-5 is configured with all 32 nodes in a single partition, so user software was required to define the number of processors used to actually solve a linear system. We present empirical speedup data collected on the parallel Gauss-Seidel algorithm for the three power systems matrices, and we also present a detailed performance analysis using actual run times for the individual subsections of the parallel Gauss-Seidel linear solver to illustrate the efficacy of the load balancing step in the preprocessing phase and to illustrate performance bottlenecks. All timing samples are for a combination of four iterations and a single convergence check. 


\section{Node Program}

$\epsilon \leftarrow \infty$

while $\epsilon>\epsilon_{\text {converge }}$

for $k=1$ to $n_{\text {iter }}$

$/{ }^{*}$ solve for $\mathbf{x}^{(k+1)}$ in the diagonal blocks */

for all rows $i$ on this processor

$\tilde{x}_{i} \leftarrow x_{i}$

$x_{i} \leftarrow b_{i}$

for each $j \in[1, n]$ such that $a_{i j} \neq 0$

endfor $x_{i} \leftarrow x_{i}-\left(a_{i j} * x_{j}\right)$

$x_{i}-x_{i} / a_{i i}$

endfor

$/^{*}$ calculate $\mathbf{L}_{m, i}^{-1} \mathbf{x}_{i}^{(k+1) * /}$

for all rows $i$ on this processor

$\tilde{x}_{i} \leftarrow x_{i}$

$\hat{b}_{i} \leftarrow b_{i}$

endfor

for all lower border non-zero rows $i$

for each $j$ such that $a_{i j} \neq 0$

endfor

$\sigma \leftarrow \sigma-\left(a_{i j} * x_{j}\right)$

using active message rpc on processor $\rho(i)$

endfor

$$
\hat{b}_{i} \leftarrow \hat{b}_{i}-\sigma
$$

$/^{*}$ solve for $\hat{\mathbf{x}}^{(k+1)}$ in the last diagonal block */

for all colors on this processor $c$

for all rows $i$ in color $c$

$x_{i} \leftarrow \hat{b}_{i}$

for each $j$ such that $a_{i j} \neq 0$

endfor

$$
x_{i} \leftarrow x_{i}-\left(a_{i j} * x_{j}\right)
$$

$x_{i} \leftarrow x_{i} / a_{i i}$

using active message rpc

broadcast $x_{i}$

endfor

wait until all values of $x_{i}$ have arrived endfor

endfor

$/^{*}$ check convergence $* /$

$\epsilon_{\lambda} \leftarrow 0$

for all rows $i$ on this processor

$\epsilon_{\lambda} \leftarrow \epsilon_{\lambda}+a b s\left(\tilde{x}_{i}-x_{i}\right)$

endfor

$\epsilon \leftarrow \epsilon_{\lambda}$

for all other processors $\rho$

using active message rpc on processor $\rho$

$\epsilon \leftarrow \epsilon+\epsilon_{\lambda}$

endfor

endwhile

Figure 4: Parallel Sparse Gauss-Seidel Algorithm
RELATIVE SPEEDUP FOR GAUSS SEIDEL

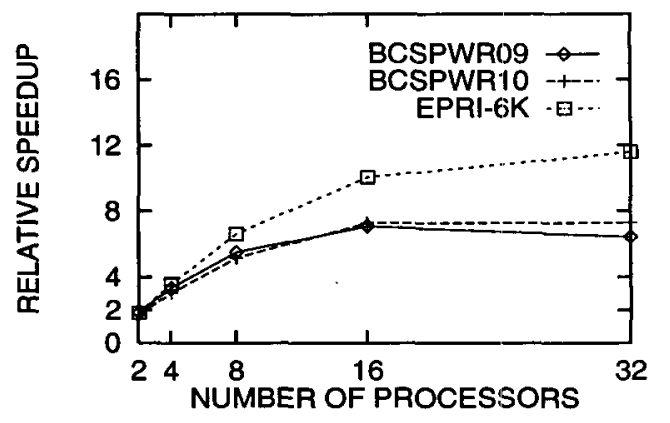

Figure 5: Relative Speedup $-2,4,8,16$, and 32 processors

\subsection{Performance Analysis}

As an introduction to the performance of the parallel Gauss-Seidel algorithm, we present a graph that plots relative speedup versus the number of processors. Figure 5 plots the best speedup measured for each of the power systems matrices for $2,4,8,16$, and 32 processors. These graphs show that performance for the EPRI-6K data set is the best of the three data sets examined. Speedup reaches a maximum of 11.6 for 32 processors and speedups of greater than 10.0 were measured for 16 processors.

Relative speedups for the BCSPWR09 and BCSPWR10 matrices are less than for the EPRI-6K matrix, but each has speedup in excess of 7.0 for 16 processors. For both the BCSPWR09 and BCSPWR10 matrices, the last diagonal block requires approximately $5 \%$ of the total calculations while the last block of the EPRI-6K matrix can be ordered so that only $1 \%$ of all calculations occur there. The likely cause for limited speedup with the Boeing-Harwell matrices is that communications overhead becomes a significant part of the overall processing time because $\hat{\mathbf{x}}^{(k+1)}$ values must be broadcast to other processors before processing can proceed to the next color. There are insufficient parallel operations when solving for $\mathbf{x}^{(k+1)}$ in the diagonal blocks for these matrices to offset the effect of the communications overhead encountered in the last block.

A detailed examination of relative speedup is presented in figure 6 for the EPRI-6K data. This figure contains a graph with four curves plotting relative speedup for each of four maximum matrix partition sizes, 128, 192, 256, and 320 nodes, used in the node- 


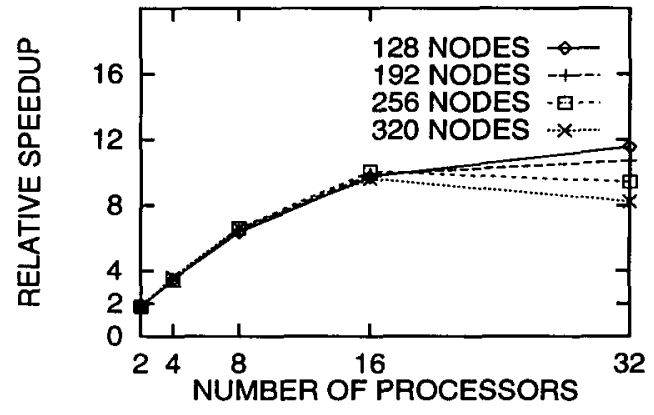

Figure 6: Relative Speedup for EPRI-6K Data - 2, $4,8,16$, and 32 processors

tearing algorithm. The speedup curves for the various matrix orderings clearly illustrate the effects of load imbalance for some matrix orderings. For all four matrix orderings, speedup is nearly equal for 2 through 16 processors. However, the values for relative speedup diverge for 32 processors.

We can look further into the cause of the disparity in the relative speedup values in the EPRI- $6 \mathrm{~K}$ data by examining the performance of each of the four distinct sections of the parallel algorithm. Figure 7 contains four graphs that each have four curves that plot processing time in milliseconds versus the number of processors for each of four values of $\max _{D B}$. These graphs are $\log$-log scaled, so for perfect speedup, processing times should fall on a straight line with decreasing slope for repeated doubling of the number of processors. One or more curves on each of the performance graphs for the diagonal blocks and upper border, for updating the last diagonal block, and for convergence checks illustrate nearly perfect speedup with as many as 32 processors. Unfortunately the performance for calculating values in the last block does not also have stellar parallel performance.

The performance graph for the diagonal blocks and lower border clearly illustrates the causes for the load imbalance observed in the relative speedup graph in figure 6. For some matrix orderings, load balancing is not able to divide the work evenly for larger numbers of processors. This occurs for larger values of $\max _{D B}$. Selecting small values of $\max _{D B}$ will provide better speedups for sixteen or more processors. Updating the last block requires both calculations of sparse (matrix $\times$ vector) products and irregular communications, but yields good performance even for 32
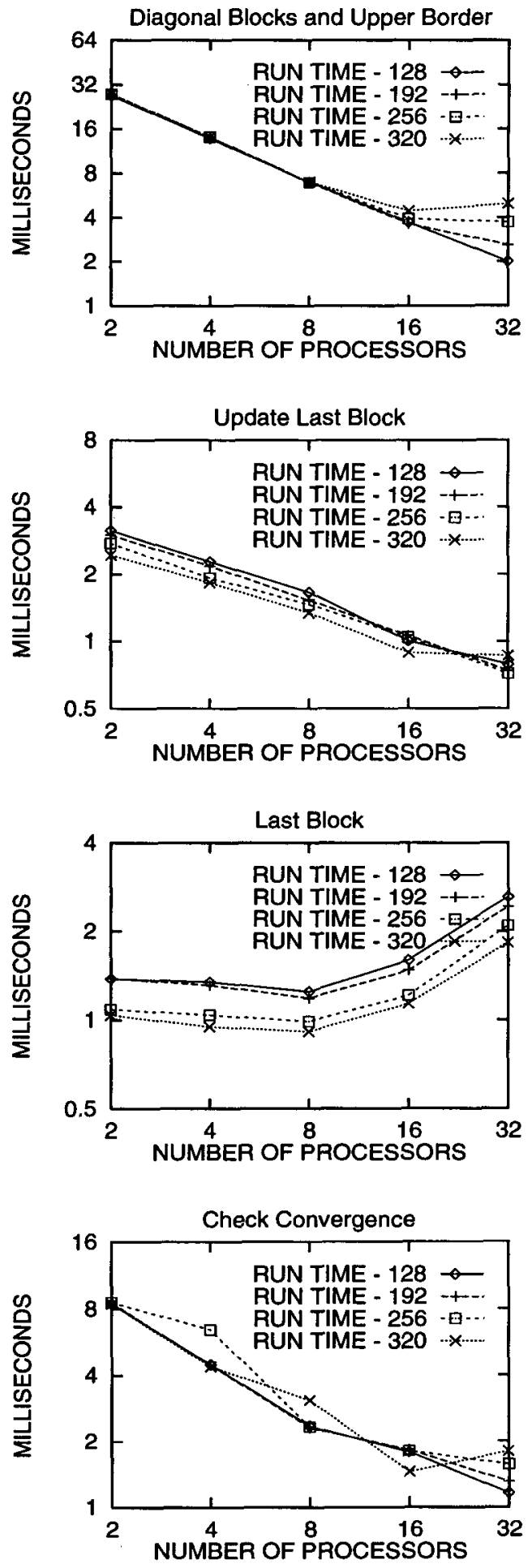

Figure 7: Timings for Algorithm Components EPRI-6K Data - 2, 4, 8, 16, and 32 processors 
processors. Update times are correlated to the size of the last diagonal block, which is inversely related to the magnitude of $\max _{D B}$. The performance graph for checking convergence illustrates that the load balancing step does not assign equal numbers of rows to all processors. The number of rows on a processor varies as a function of the load balancing. While the curves on this graph are somewhat erratic, performance is improving with near perfect parallelism even for 32 processors.

We must reiterate that all available parallelism in this work is a result of ordering the matrix and identifying relationships in the connectivity pattern within the structure of the matrix. Power systems load flow matrices are some of the most sparse irregular matrices encountered. For the EPRI-6K data, the most frequently encountered number of edges at a node is only two, and $84.4 \%$ of the nodes have three or less edges. For the BCSPWR 10 matrix, $71 \%$ of the nodes have three or less edges. Consequently, power systems matrices pose significant challenges to produce efficient parallel sparse matrix algorithms.

In figure 8 , we present a representative ordering of the EPRI-6K data with $\max _{D B}$ equal to 256 nodes. This matrix represents the adjacency structure of the network graph, and clearly illustrates sparsity. Nonzero entries in the matrix are represented as dots, and the matrix is delimited by a bounding box. This figure contains two matrices: the ordered sparse matrix and an enlargements of the last block after multi-coloring. This partitioned matrix has been load-balanced for eight processors. The number of nodes in the last diagonal block is 120 , the numbers of edges are only 22 , and this matrix partition is bipartite - requiring only two colors.

To obtain the full benefits of parallel processing speedup throughout a load flow application, all data redistribution must be eliminated. Jacobian calculations when solving the systems of non-linear equations must consider the processor/data assignments from the sparse linear solver. Otherwise, data redistribution overhead would nullify any speedup obtainable in the parallel linear solver.

\subsection{Convergence}

Convergence for a given data set is critical to the performance of an iterative linear solver. We have applied our solver to sample positive definite matrices that have actual power networks as the basis for the sparsity pattern, and random values for the entries. A sample of measured convergence data is presented in table 1 . This table presents the total error and

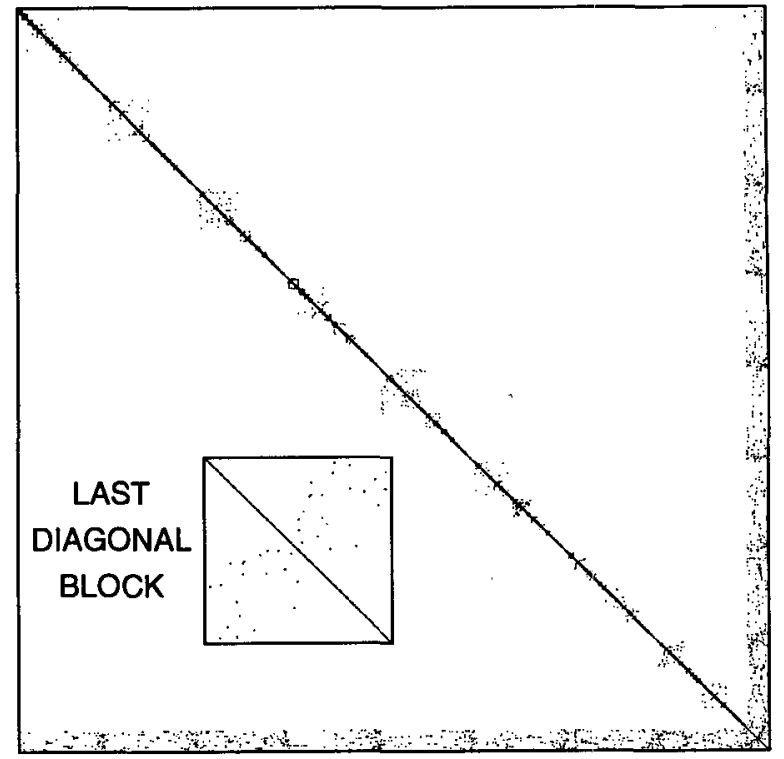

Figure 8: Ordered EPRI-6K Matrix - $\max _{D B}=256$

\begin{tabular}{|c|c|c|}
\hline & Total Error & \\
Iteration & $\sum_{\forall i} a b s\left(x_{i}^{(k+1)}-x_{i}^{(k)}\right)$ & $\max _{\forall i} x_{i}^{(k+1)}$ \\
\hline 1 & 0.983654764216 & 0.000478248320 \\
\hline 2 & 0.000143661684 & 0.000478321438 \\
\hline 3 & 0.000000018556 & 0.000478321442 \\
\hline 4 & 0.000000000002 & 0.000478321442 \\
\hline
\end{tabular}

Table 1: Convergence for EPRI-6K Data

the maximum value for an iteration. All initial values, $\mathbf{x}^{(0)}$, have been defined to equal 0.0. Convergence is rather rapid, and after four iterations, total error equals $2 \times 10^{-12}$. We hypothesize that this good convergence rate is in part due to having good estimates of the initial starting vector. For actual solutions of power systems load flows, this solver would be used within an iterative non-linear solver, so good estimates of starting points for each solution would be readily available.

\section{Conclusions}

We have developed a parallel sparse Gauss-Seidel solver with the potential for good relative speedup for the very sparse, irregular matrices encountered in electrical power system applications. Block-diagonal- 
bordered matrix structure offers promise for simplified implementation and also offers a simple decomposition of the problem into clearly identifiable subproblems. The node-tearing ordering heuristic has proven to be successful in identifying the hierarchical structure in the power systems matrices, and reducing the number of coupling equations so that the graph multi-coloring algorithm can usually color the last block with only two or three colors. All available parallelism in our Gauss-Seidel algorithm is derived from within the actual interconnection relationships between elements in the matrix, and identified in the sparse matrix orderings. Consequently, available parallelism is not unlimited. Relative speedup tends to increase nicely until either load-balance overhead or communications overhead cause speedup to level off.

We have shown that, depending on the matrix, relative efficiency declines rapidly after 8 or 16 processors, limiting the utility of applying large numbers of processors to a single parallel linear solver. Nevertheless, other dimensions exist in electrical power system applications that can be exploited to use large numbers of processors efficiently. While a moderate number of processors can be efficiently applied to a single power system simulation, multiple events can be simulated simultaneously.

\section{Acknowledgments}

We thank Alvin Leung, Nancy McCracken, Paul Coddington, and Tony Skjellum for their assistance in this research. This work has been supported in part by Niagara Mohawk Power Corporation, the New York State Science and Technology Foundation, the NSF under co-operative agreement No. CCR-9120008, and ARPA under contract \#DABT63-91-K-0005.

\section{References}

[1] D. Brélaz. New Methods to Color the Vertices of a Graph. Comm. ACM, 22:251, 1979.

[2] I. S. Duff, R. G. Grimes, and J. G. Lewis. Users Guide for the Harwell-Boeing Sparse Matrix Collection. Technical report, Boeing Computer Services, 1992.

[3] Electrical Power Research Institute, Palo Alto, California. Extended Transient-Midterm Stability Program: Version 3.0 - Volume 4: Programmers Manual, Part 1, April 1993.
[4] G. Fox, M. Johnson, G. Lyzenga, S. Otto, J. Salmon, and D. Walker. Solving Problems on Concurrent Processors. Prentice Hall, 1988.

[5] G. Golub and J. M. Ortega. Scientific Computing with an Introduction to Parallel Computing. Academic Press, Boston, MA., 1993.

[6] M. T. Heath, E. Ng, and B. W. Peyton. Parallel Algorithms for Sparse Linear Systems. In Parallel Algorithms for Matrix Computations, pages 83124. SIAM, Philadelphia, 1991.

[7] G. Huang and W. Ongsakul. Managing the Bottlenecks in Parallel Gauss-Seidel Type Algorithms for Power Flow Analysis. Proceedings of the 18th Power Industry Computer Applications (PICA) Conference, pages 74-81, May 1993.

[8] D. P. Koester, S. Ranka, and G. C. Fox. A Parallel Gauss-Seidel Algorithm for Sparse Power Systems Matrices. Technical Report SCCS-630, NPAC, April 1994.

[9] D. P. Koester, S. Ranka, and G. C. Fox. Parallel Block-Diagonal-Bordered Sparse Linear Solvers for Electrical Power System Applications. In Proceeding of the Scalable Parallel Libraries Conference. IEEE Press, 1994.

[10] D. P. Koester, S. Ranka, and G. C. Fox. Parallel Choleski Factorization of Block-DiagonalBordered Sparse Matrices. Technical Report SCCS-604, NPAC, January 1994.

[11] R. A. Saleh, K. A. Gallivan, M. Chang, I. N. Hajj, D. Smart, and T. N. Trick. Parallel Circuit Simulation on Supercomputers. Proceedings of the IEEE, 77(12):1915-1930, December 1989.

[12] A. Sangiovanni-Vincentelli, L. K. Chen, and L. O. Chua. Node-Tearing Nodal Analysis. Technical Report ERL-M582, Electronics Research Laboratory, College of Engineering, University of California, Berkeley, October 1976.

[13] T. von Eicken, D. E. Culler, S. C. Goldstein, and K. E. Schauser. Active Messages: a Mechanism for Integrated Communication and Computation. In Nineteenth International Symposium on Computer Architecture, New York, 1992. ACM Press.

[14] Y. Wallach. Calculations and Programs for Power System Networks. Prentice-Hall, 1986. 